\title{
Array-based karyotyping in chronic lymphocytic leukemia (CLL) detects new unbalanced abnormalities that escape conventional cytogenetics and CLL FISH panel
}

\author{
Helena Urbankova, Tomas Papajik, Radek Plachy, Milena Holzerova, Jana Balcarkova, Martina Divoka, Vit Prochazka, \\ Zuzana Pikalova, Karel Indrak, Marie Jarosova
}

\begin{abstract}
Aims. Chronic lymphocytic leukemia (CLL) is the most common adult leukemia with a very heterogeneous course. Progress in molecular genetic characterization of CLL has confirmed the prognostic role of unbalanced chromosomal abnormalities currently defined by molecular cytogenetic methods: conventional karyotyping and FISH. However, a significant percentage of genomic abnormalities escapes routine investigation due to the limitations of these methods. It is presently clear that some of these aberrations have impact on prognosis and disease progression.

Methods. We examined copy number changes in the tumor genomes of $50 \mathrm{CLL}$ patients using bacterial artificial chromosome (BAC) and/or oligonucleotide array platforms.

We compared the results of arrayCGH with those obtained by FISH and conventional cytogenetics and evaluated their clinical importance.

Results. A total of 111 copy number changes were detected in 43 patients (86\%) with clonal abnormalities present in at least $23 \%$ of the cells. Moreover, 14 patients (28\%) were found to have 39 genomic changes that had not been detected by standard cytogenetic and/or FISH analyses. These included possibly prognostically important recurrent $2 p$ and 8q24 gains. The most frequent unbalanced changes involved chromosomes 18, 7, 3, 9 and 17. We also determined the minimal deleted region on chromosome $6 q$ in 7 cases by chromosome 6/7 specific array.

Conclusions. The results showed that a subset of potentially significant genomic aberrations in CLL is being missed by the current routine techniques. Further, we clearly demonstrated the robustness, high sensitivity and specificity of the arrayCGH analysis as well as its potential for use in routine screening of CLL.
\end{abstract}

Key words: chronic lymphocytic leukemia, cancer, arrayCGH, copy number changes, chromosomal abnormalities, $\operatorname{del}(6)(q)$

Received: November 8, 2011; Accepted: February 27, 2012; Available online: June 1, 2012 http://dx.doi.org/10.5507/bp.2012.031

Department of Hemato-oncology, University Hospital Olomouc and Faculty of Medicine and Dentistry, Palacky University Olomouc, Czech Republic

Corresponding author: Helena Urbankova, e-mail:hpospis@centrum.cz

\section{INTRODUCTION}

B-cell chronic lymphocytic leukemia is the most common adult leukemia in the Western world. The clinical course of CLL is heterogeneous. Some patients with CLL survive for many years without therapy, showing minimal signs and symptoms throughout the course of the disease. They have a survival time similar to age-matched controls. Other patients, however, have rapidly deteriorating blood counts and organomegaly, leading to death. The Rai and Binet clinical staging systems $\mathrm{s}^{1,2}$ were developed to estimate the prognosis of CLL patients but are unable to predict a poor prognosis at early stages of the disease. Recently, there has been an active interest in identifying molecular and cytogenetic markers useful for predicting the clinical course in these patients. Among others, the prognostic role of $I g V H$ mutational status and expression of CD38 and ZAP-70 was established. A subset of genomic aberrations was identified as important independent predic- tors of disease progression and survival ${ }^{3}$. Deletions of $11 \mathrm{q}$ and $17 \mathrm{p}$, affecting the ataxia telangiectasia mutated (ATM) gene and p53 tumor suppressor gene (TP53) respectively, have been associated with disease progression and reduced survival ${ }^{47}$. Early identification of these deletions could allow clinicians to choose appropriate therapy for these patients ${ }^{8,9}$.

Other recurrent aberrations are detected in CLL. Trisomy 12 was described as the first recurrent chromosome aberration in CLL by Gahrton et al. in 1980 $\left(\right.$ ref. $\left.{ }^{10}\right)$. This aberration initially correlated with an advanced stage of the disease and shorter survival time ${ }^{11}$. Deletion of 13q14, likely to target mir-15 and mir-16 (ref. ${ }^{12}$ ), is the most common cytogenetic abnormality in CLL correlated with a favorable prognosis in the absence of other abnormalities ${ }^{13}$. Additionally, deletion of $6 \mathrm{q}$ is observed in approximately $6 \%$ of CLL patients. While Oscier et al. ${ }^{14}$ reported shorter treatment-free intervals for CLL patients with a $6 \mathrm{q}$ deletion, no adverse prog- 
nostic effect was found in other large CLL studies ${ }^{15,16}$. Currently, no gene contributing to CLL pathogenesis has been identified at $6 \mathrm{q}$ even though some candidate genes located in the deleted region have been identified, such as $P I C 3 A$ and $M Y B$ (ref. ${ }^{16}$ ). Translocations and deletions of $14 \mathrm{q} 32$ involving the $I G H$ gene were found in up to $5 \%$ of CLL patients ${ }^{17-19}$. Other chromosomal abnormalities involving regions of $3 q, 8 q, 9 p$ and other regions are less frequent in CLL. Therefore, routine diagnostic investigation of CLL patients includes conventional cytogenetics and interphase FISH analysis; a panel of 4-6 probes was designed to analyze the $11 q 22,12,13 q 14 / 13 q 34,17 p 13$, $6 q 21$ and $14 q 32$ regions as these regions are recurrently targeted by numerical and structural aberrations in CLL. While cytogenetics is informative in up to $50 \%$ of CLLs, FISH detects chromosomal aberrations of the above loci in more than $80 \%$ of patients. However, the latter technique provides a very limited view of the genomic aberrations because only a number of loci are analyzed. It is presently unclear whether the CLL-associated aberrations that are not included in the standard FISH panel have any impact on prognosis and disease progression. With the advent and availability of arrayCGH technology, highresolution, locus-specific analysis and genome-wide evaluation can be combined into a single test ${ }^{20}$. To utilize the ability of arrayCGH, we studied 50 CLL patients recently diagnosed and treated at our institution. This study aimed at comparing arrayCGH results with conventional cytogenetics and FISH and identifying possible unbalanced chromosomal aberrations that escaped banding and FISH analysis. Moreover, using the tiling path chromosome 6 arrayCGH platform, we mapped $6 \mathrm{q}$ deletions in selected CLL patients and defined the smallest commonly deleted region likely to harbor the CLL-related tumor suppressor gene. Another aim of this pilot study was to demonstrate the potential clinical utility of arrayCGH method.

\section{MATERIALS AND METHODS}

\section{Patients}

A total of 34 male and 16 female patients with a median age of 61 years were diagnosed with CLL and treated at the Department of Hemato-oncology, University Hospital Olomouc, Czech Republic. Peripheral blood was examined at the time of diagnosis or during the course of the disease after obtaining informed consent. Of the patients, 30 were examined at the time of diagnosis while the remaining 20 were examined during the course of the disease. All patients of the latter group were treated with different chemotherapy regimens, such as CHOP (cyclophosphamide, doxorubicin, vincristine and prednisone), R-CHOP (rituximab-CHOP), and FLUCY (fludarabine and cyclophosphamide).

Overall, 18 patients were Binet A, 3 patients were Binet A/B, 16 were Binet B and 13 were Binet C (Table 1). The median overall survival (OS) was 37.3 months (range 12.5-215.9). A total of 43 patients are alive with a median OS of 37.9 months.

\section{Conventional cytogenetics and FISH}

Cytogenetic examinations were performed on metaphases obtained by parallel short-term culture in the presence of 12-O-tetradecanoylphorbol-13-acetate (TPA) (Sigma-Aldrich, Munich, Germany), the immunostimulatory CpG-oligonucleotide DSP30 (TIBMolbiol, Berlin, Germany) and interleukin-2 (IL-2) (Sigma-Aldrich). G-banding analysis as well as fluorescence in situ hybridization (FISH) were performed as described in standard procedures.

The probes used for FISH analysis included LSI D13S319(13q14)/13q34/CEP12, LSI p53, LSI ATM, LSI IGH, LSI ALK, LSI NMYC, LSI MYC, CEP 2, 11, 17, 18 (Abbott Molecular Inc, Des Plaines, USA), ON MM 15q22/6q21, GLI(12q13) (Kreatech Diagnostics, Amsterdam, Netherlands), XCAP 2 (MetaSystems, Altussheim, Germany) and BAC derived probes RP11-373L24 and RP11-498O5 covering the REL locus (BlueGnome, Cambridge, UK). At least 200 nuclei were analyzed, and the number of abnormal nuclei was expressed as a percentage of the scored nuclei. The cutoff level for reliable determination of abnormality was established as the presence of at least $2.5 \%$ of abnormal nuclei. In patients with a complex karyotype, WCP probes (Cambio, Cambridge, UK; MetaSystems) and MFISH (MetaSystems) were used.

\section{ArrayCGH analysis}

DNA for arrayCGH examination was isolated by the standard phenol-chloroform method from the samples taken at the same time as samples for cytogenetic and FISH examination.

Three different BAC arrayCGH platforms with a resolution of approximately $1 \mathrm{Mb}$ were used. The most frequently used arrayCGH chip (20 patients) was manufactured by LUMC, Leiden, Netherlands. A $1 \mathrm{Mb}$ BAC array platform (VIB MicroArray Facility, Catholic University Leuven, Leuven, Belgium) was used for 12 patients. For other 12 patients, arrayCGH analysis was performed using CytoChip_Haem_v0.1 (BlueGnome). Finally, the commercially available oligonucleotide 4 x44K array (Agilent Technologies, Santa Clara, USA) was utilized for 6 patients. These 4 platforms were compared to each other using hybridisation with control male and female sample. The bias detected across platforms was in all cases under the detection limits defined for this study.

The tiling path chromosome 6 array was constructed by VIB MicroArray Facility, Leuven, Belgium and was used for 7 patients with detected $\operatorname{del}(6)(q)$. The arrayCGH experiments followed the standard protocols and manufacturers' recommendations ${ }^{21-24}$. Hybridized microarrays were scanned with the Axon 2400A microarray scanner, and the results were evaluated according to microarray types. The microarrays manufactured in Leuven and Leiden were evaluated using a homemade spreadsheet application; microarrays manufactured by BlueGnome were analyzed with the BlueFuse software (Bluegnome) and the Agilent oligo 4x44K array was analyzed with the Agilent Feature Extraction Software (Agilent Technologies). 
Table 1. Clinical, cytogenetic and FISH findings in 50 CLL patients.

\begin{tabular}{|c|c|c|c|c|c|c|c|c|c|c|}
\hline \multirow{2}{*}{ No. } & \multirow{2}{*}{$\begin{array}{l}\text { Sex/ } \\
\text { Age }\end{array}$} & \multirow{2}{*}{$\begin{array}{l}\text { Binet } \\
\text { stage }\end{array}$} & \multirow{2}{*}{$\begin{array}{l}\text { Mutation } \\
\text { status } \\
\text { IgVH }\end{array}$} & \multirow{2}{*}{ ISCN Cytogenetics } & \multicolumn{6}{|c|}{ FISH } \\
\hline & & & & & TP53 & ATM & c12 & $13 q 14$ & $6 q 21$ & IGH \\
\hline 1 & $\mathrm{M} / 62$ & B & UM & UNS & $\mathrm{N}$ & $\mathrm{N}$ & $\mathrm{N}$ & $\mathrm{N}$ & ND & $\mathrm{N}$ \\
\hline 2 & $\mathrm{M} / 50$ & B & M & $48 \sim 49, \mathrm{XY}, ?+19, ?+20,1-2 \operatorname{mar}[\mathrm{cp} 4] / 46, \mathrm{XY}[2]$ & $\mathrm{N}$ & $\mathrm{N}$ & $\mathrm{N}$ & D81\% & ND & ND \\
\hline 3 & $\mathrm{~F} / 52$ & $\mathrm{C}$ & M & 46,XY,t(3;14)(q?;q?)[9]/46,XY[1] & $\mathrm{N}$ & $\mathrm{N}$ & $\mathrm{N}$ & $\mathrm{N}$ & $\mathrm{N}$ & $\mathrm{R} 64 \%$ \\
\hline 4 & M70 & $\mathrm{C}$ & UM & UNS & $\mathrm{N}$ & $\mathrm{D} 22 \%$ & $\mathrm{~N}$ & D96\% & ND & D5'95\% \\
\hline 5 & $\mathrm{M} / 60$ & $\mathrm{C}$ & $\mathrm{UM}$ & $\begin{array}{l}\text { 46,XY,del(6)(q?),der(7)t(7;12)(q3?6;q?12q?ter), } \\
? \operatorname{del}(13)(\mathrm{q})[12] / 46, \mathrm{XY}[1]\end{array}$ & $\mathrm{N}$ & $\mathrm{N}$ & $\mathrm{N}$ & D84\% & ND & D5’80\% \\
\hline 6 & $\mathrm{M} / 57$ & $\mathrm{~B}$ & M & $46, \mathrm{XY}[5]$ & $\mathrm{N}$ & $\mathrm{N}$ & $\mathrm{N}$ & D48\% & ND & ND \\
\hline 7 & $\mathrm{M} / 74$ & A & M & $45 \sim 46, X,-Y, ?-9, ?-19,+3 \operatorname{mar}[\mathrm{cp} 3] / 46, \mathrm{XY}[9]$ & $\mathrm{N}$ & $\mathrm{N}$ & $\mathrm{N}$ & D88\% & ND & $\mathrm{N}$ \\
\hline 8 & M46 & $\mathrm{B}$ & UM & $\begin{array}{l}\text { 46,XY,der(11),der(13)[10].ish t(11;13)(q?21;q?) } \\
\text { ins(13;14)(q?;q?)(wcp11+,wcp13+,wcp14+)/46,XY[4] }\end{array}$ & $\mathrm{N}$ & $\mathrm{N}$ & $\mathrm{N}$ & D81\% & ND & $\mathrm{N}$ \\
\hline 9 & $\mathrm{M} / 61$ & $\mathrm{C}$ & UM & $45, \mathrm{XY},-15,-17,+\operatorname{mar}[9]$ & $\mathrm{N}$ & D81\% & $\mathrm{N}$ & $\mathrm{N}$ & ND & $\mathrm{N}$ \\
\hline 10 & $\mathrm{~F} / 71$ & $\mathrm{~B}$ & UM & $46, \mathrm{XX}[5]$ & $\mathrm{N}$ & $\mathrm{N}$ & $\mathrm{N}$ & $\mathrm{N}$ & ND & $\mathrm{N}$ \\
\hline 11 & $\mathrm{M} / 42$ & $\mathrm{~B}$ & UM & UNS & $\mathrm{N}$ & $\mathrm{N}$ & $\mathrm{N}$ & D39\% & ND & $\mathrm{N}$ \\
\hline 12 & $\mathrm{M} / 56$ & $\mathrm{C}$ & UM & UNS & $\mathrm{N}$ & $\mathrm{N}$ & $\mathrm{N}$ & $\mathrm{N}$ & ND & $\mathrm{N}$ \\
\hline 13 & $\mathrm{M} / 72$ & B & M & $46, X Y[21]$ & $\mathrm{N}$ & $\mathrm{N}$ & $\mathrm{N}$ & D79\% & ND & $\mathrm{N}$ \\
\hline 14 & $\mathrm{~F} / 42$ & A & M & $46, \mathrm{XX}[30]$ & $\mathrm{N}$ & $\mathrm{N}$ & $\mathrm{N}$ & D94\% & ND & D5’87\% \\
\hline 15 & $\mathrm{M} / 51$ & A & UM & $45, \mathrm{XY}, ? \operatorname{der}(1), \operatorname{del}(6)(\mathrm{q}),-13[16]$ & $\mathrm{N}$ & $\mathrm{N}$ & $\mathrm{N}$ & D75\% & D78\% & D5’85\% \\
\hline 16 & $\mathrm{M} / 66$ & $\mathrm{C}$ & UM & UNS & $\mathrm{N}$ & $\mathrm{N}$ & $\mathrm{N}$ & $\mathrm{N}$ & $\mathrm{D} 83 \%$ & $\mathrm{~N}$ \\
\hline 17 & $\mathrm{M} / 63$ & A & UM & $\begin{array}{l}47 \sim 47, \mathrm{XY}, \operatorname{der}(3), ? \operatorname{der}(4), ?-5,-7, \operatorname{der}(11),+12, ? \operatorname{der}(14), \\
+2-3 \operatorname{mar}[\operatorname{cp} 11]\end{array}$ & $\mathrm{N}$ & D77\% & $+74 \%$ & $\mathrm{~N}$ & $\mathrm{~N}$ & $\mathrm{~N}$ \\
\hline 18 & $\mathrm{~F} / 63 \dagger$ & $\mathrm{B}$ & UM & ND & D97\% & $\mathrm{N}$ & $\mathrm{N}$ & D87\% & $\mathrm{N}$ & $\mathrm{N}$ \\
\hline 19 & $\mathrm{M} / 58$ & $\mathrm{~B}$ & UM & $46, X Y[13]$ & $\mathrm{N}$ & $\mathrm{N}$ & $\mathrm{N}$ & D88\% & D89\% & $\mathrm{N}$ \\
\hline 20 & $\mathrm{M} / 70$ & A & M & $46, X Y[15]$ & D84\% & $\mathrm{N}$ & $\mathrm{N}$ & D82\% & $\mathrm{N}$ & ND \\
\hline 21 & $\mathrm{~F} / 71$ & $\mathrm{C}$ & $\mathrm{UM}$ & $\begin{array}{l}\text { 46,XX, der(1),?der(2), der(8), der(11)t(11;?),?der(14) } \\
\text { [cp9] }\end{array}$ & $\mathrm{N}$ & $\mathrm{D} 83 \%$ & $\mathrm{~N}$ & $\mathrm{~N}$ & $\mathrm{~N}$ & $\mathrm{~N}$ \\
\hline 22 & $\mathrm{M} / 78$ & A & M & $44 \sim 45, \mathrm{XY}, \mathrm{r}(12)(\mathrm{p} 13 \mathrm{q} 24.3)$, inc [cp3]/46,XY[4] & $\mathrm{D} 43 \%$ & $\mathrm{~N}$ & $\mathrm{~N}$ & D78\% & $\mathrm{N}$ & D5’47\% \\
\hline 23 & $\mathrm{M} / 58 \dagger$ & A & UM & $46, X Y, \operatorname{del}(11)(\mathrm{q} ?)[6] / 46, \mathrm{XY}[5]$ & $\mathrm{N}$ & D91\% & $\mathrm{N}$ & D76\% & $\mathrm{N}$ & $\mathrm{N}$ \\
\hline 24 & $\mathrm{~F} / 60$ & A & M & UNS & $\mathrm{N}$ & $\mathrm{N}$ & $\mathrm{N}$ & D94\% & $\mathrm{N}$ & $\mathrm{N}$ \\
\hline 25 & $\mathrm{M} / 46 \dagger$ & $\mathrm{B}$ & UM & 46,XY[4] & $\mathrm{N}$ & D88\% & $\mathrm{N}$ & D86\% & $\mathrm{N}$ & $\mathrm{N}$ \\
\hline 26 & $\mathrm{M} / 73$ & A & M & $46, X Y[13]$ & $\mathrm{N}$ & $\mathrm{N}$ & $+43 \%$ & D43\% & $\mathrm{N}$ & $\mathrm{N}$ \\
\hline 27 & $\mathrm{M} / 70$ & $\mathrm{C}$ & UM & UNS & $\mathrm{N}$ & D94\% & $\mathrm{N}$ & D91\% & $\mathrm{N}$ & D5'93\% \\
\hline 28 & $\mathrm{~F} / 70$ & $\mathrm{~A} / \mathrm{B}$ & $\mathrm{UM}$ & UNS & $\mathrm{N}$ & $\mathrm{N}$ & $+46 \%$ & $\mathrm{~N}$ & $\mathrm{~N}$ & D3'53\% \\
\hline 29 & $\mathrm{M} / 68 \dagger$ & $\mathrm{C}$ & UM & $46, \mathrm{XY}[5]$ & D93\% & $\mathrm{N}$ & $\mathrm{N}$ & D93\% & ND & D3'97\% \\
\hline 30 & $\mathrm{M} / 83$ & $\mathrm{~A} / \mathrm{B}$ & $\mathrm{UM}$ & 46,XY,del(14q)[8] & $\mathrm{N}$ & $\mathrm{N}$ & $\mathrm{N}$ & $\mathrm{N}$ & $\mathrm{N}$ & D60\% \\
\hline 31 & $\mathrm{M} / 60$ & $\mathrm{C}$ & $\mathrm{UM}$ & $\begin{array}{l}\text { 46,XY,del(11)(q?)[5]/46,XY,?der(1), } \operatorname{del}(11)(\mathrm{q} ?), \\
\text { ?der(17)[4]/46,XY[2] }\end{array}$ & $\mathrm{N}$ & D53\% & $\mathrm{N}$ & D93\% & $\mathrm{N}$ & $\mathrm{N}$ \\
\hline 32 & $\mathrm{~F} / 50$ & B & UM & $46, \mathrm{XX}, \operatorname{der}(13), \operatorname{der}(16)[3] / 45 \sim 46, \mathrm{XX},-19,+\operatorname{mar}[\mathrm{cp} 5]$ & $\mathrm{N}$ & $\mathrm{N}$ & $\mathrm{N}$ & $\mathrm{N}$ & D60\% & UNS \\
\hline 33 & $\mathrm{~F} / 52$ & $\mathrm{~A}$ & UM & $46, X Y, \operatorname{del}(11 \mathrm{q})[10]$ & $\mathrm{N}$ & D76\% & $\mathrm{N}$ & $\mathrm{N}$ & $\mathrm{N}$ & $\mathrm{N}$ \\
\hline 34 & $\mathrm{~F} / 56$ & A & UM & $46, \mathrm{XX}[14]$ & $\mathrm{N}$ & $\mathrm{N}$ & $\mathrm{N}$ & D92\% & $\mathrm{N}$ & UNS \\
\hline 35 & $\mathrm{M} / 47$ & $\mathrm{~B}$ & UM & $46, \mathrm{XY}[4]$ & $\mathrm{N}$ & $\mathrm{N}$ & $\mathrm{N}$ & D77\% & $\mathrm{N}$ & $\mathrm{N}$ \\
\hline 36 & $\mathrm{M} / 60$ & A & UM & UNS & $\mathrm{N}$ & $\mathrm{D} 48 \%$ & $\mathrm{~N}$ & D45\% & $\mathrm{D} 50 \%$ & UNS \\
\hline 37 & $\mathrm{~F} / 52$ & $\mathrm{~A} / \mathrm{B}$ & M & $46, X X, \operatorname{del}(13 q)[5] / 46, X X[10]$ & $\mathrm{N}$ & $\mathrm{N}$ & $\mathrm{N}$ & D92\% & $\mathrm{N}$ & $\mathrm{N}$ \\
\hline 38 & $\mathrm{M} / 65$ & $\mathrm{~B}$ & UM & $47, \mathrm{XY},+12[29] / 46, \mathrm{XY}[1]$ & $\mathrm{N}$ & $\mathrm{N}$ & $+80 \%$ & $\mathrm{~N}$ & $\mathrm{~N}$ & $\mathrm{~N}$ \\
\hline 39 & $\mathrm{M} / 59$ & $\mathrm{C}$ & UM & $\begin{array}{l}\text { 45,XY,der(2)dup(2)(p13)ins(2;13)(p?;?q),der(11) } \\
\text { t(11;17)(q?24;?p),der(13)t(2;13)(p?;q?13),?dic(17;22) } \\
(\mathrm{p} 11 ; \mathrm{p} 11)[5] / 45, \mathrm{X}, \operatorname{der}(\mathrm{Y}) \mathrm{t}(\mathrm{Y} ; 15)(\mathrm{q} ? 11 ; \mathrm{q} ? 21), \operatorname{idem}[11]\end{array}$ & $\mathrm{D} 85 \%$ & $\mathrm{~N}$ & $\mathrm{~N}$ & $\mathrm{~N}$ & $\mathrm{~N}$ & $\mathrm{~N}$ \\
\hline 40 & $\mathrm{M} / 68 \dagger$ & $\mathrm{B}$ & $\mathrm{UM}$ & UNS & D55\% & $\mathrm{N}$ & $\mathrm{N}$ & D97\% & $\mathrm{N}$ & $\mathrm{N}$ \\
\hline 41 & $\mathrm{~F} / 65$ & A & $\begin{array}{l}\text { boundary } \\
\text { value }\end{array}$ & 46,XX,der(7)t(2;7)(p?;q?36),del(13q)[cp9] & $\mathrm{N}$ & $\mathrm{N}$ & $\mathrm{N}$ & D95\% & $\mathrm{N}$ & $\mathrm{N}$ \\
\hline 42 & $\mathrm{~F} / 71$ & A & $\mathrm{UM}$ & $\begin{array}{l}46, X X, \operatorname{der}(11) \operatorname{del}(11)(\mathrm{q} ?) \mathrm{t}(11 ; 20)(\mathrm{q} ? ; ?), \operatorname{der}(16) \\
\mathrm{t}(16 ; 19)(? ; \mathrm{p} ?), \operatorname{der}(20) \mathrm{t}(17 ; 20)(? \mathrm{q} ; ?)[13]\end{array}$ & $\mathrm{N}$ & D90\% & $\mathrm{N}$ & $\mathrm{N}$ & $\mathrm{N}$ & $\mathrm{N}$ \\
\hline 43 & $\mathrm{M} / 61$ & A & UM & $46, X Y[30]$ & $\mathrm{N}$ & $\mathrm{N}$ & $\mathrm{N}$ & D $82 \%$ & $\mathrm{~N}$ & $\mathrm{~N}$ \\
\hline 44 & $\mathrm{M} / 54$ & $\mathrm{~B}$ & UM & $46, \mathrm{XY}[3] / 46, \mathrm{XY}, \operatorname{del}(11 \mathrm{q})[3]$ & $\mathrm{N}$ & D47\% & $\mathrm{N}$ & D94\% & $\mathrm{N}$ & D5'90\% \\
\hline 45 & $\mathrm{~F} / 77 \dagger$ & $\mathrm{C}$ & M & $47, \mathrm{XX},+12[3] / 46, \mathrm{XX}[2]$ & $\mathrm{N}$ & $\mathrm{N}$ & $+79 \%$ & $\mathrm{~N}$ & $\mathrm{~N}$ & D5'85\% \\
\hline 46 & $\mathrm{~F} / 83$ & A & M & UNS & $\mathrm{N}$ & $\mathrm{N}$ & $\mathrm{N}$ & D74\% & $\mathrm{N}$ & $\mathrm{N}$ \\
\hline 47 & $\mathrm{M} / 65$ & A & UM & $46, X Y[10]$ & $\mathrm{N}$ & $\mathrm{N}$ & $\mathrm{N}$ & D66\% & $\mathrm{N}$ & D5'80\% \\
\hline 48 & $\mathrm{M} / 50$ & $\mathrm{~A}$ & UM & UNS & $\mathrm{N}$ & D92\% & $\mathrm{N}$ & D53\% & $\mathrm{N}$ & $\mathrm{N}$ \\
\hline 49 & $\mathrm{~F} / 62$ & $\mathrm{C}$ & UM & $46, \mathrm{XX}[23]$ & $\mathrm{N}$ & $\mathrm{N}$ & $\mathrm{N}$ & D69\% & $\mathrm{N}$ & D5'63\% \\
\hline 50 & $\mathrm{M} / 52 \dagger$ & $\mathrm{B}$ & UM & $44 \sim 46, X Y,-6,-14,-17,+1-3 \operatorname{mar}[\mathrm{cp} 10]$ & $\mathrm{N}$ & $\mathrm{N}$ & $\mathrm{N}$ & $\mathrm{N}$ & D67\% & $\mathrm{N}$ \\
\hline
\end{tabular}

Abbreviations: N - normal findings; D - deletion; + - three copies of CEP 12; ND - not done; R - the rearrangement of gene; UNS - unsuccessful examination; $\uparrow$ patient died 
Table 2. ArrayCGH results in 50 CLL patients.

\begin{tabular}{|c|c|c|}
\hline No. & gains & losses \\
\hline 1 & - & - \\
\hline 2 & - & $13 q 13.1 q 14.2(33,894,709-48,999,920)$ \\
\hline 3 & - & - \\
\hline 4 & - & - \\
\hline 5 & $12 q 13.13 q 24.33(52,742,592-133,525,406)$ & 6q16.3q22.31(103,336,301-120,550,918), 13q13.3q21.33(38,155,980-71,519,829) \\
\hline 6 & - & $13 q 13.1 q 14.3(33,894,709-52,514,204)$ \\
\hline 7 & - & $13 \mathrm{q} 14.2 \mathrm{q} 21.2(47,651,918-59,886,576)$ \\
\hline 8 & - & $13 q 14.2 q 14.3(48,862,679-51,573,841), 13 q 21.31(63,104,009-64,200,914)$ \\
\hline 9 & - & $15 q 11.2 q 15.1(25,185,941-41,157,098)$ \\
\hline 10 & - & - \\
\hline 11 & - & - \\
\hline 12 & - & - \\
\hline 13 & - & $13 \mathrm{q} 14.2 \mathrm{q} 14.3(48,999,921-52,514,204)$ \\
\hline 14 & - & $13 \mathrm{q} 14.2 \mathrm{q} 14.3(50,062,881-51,573,841)$ \\
\hline 15 & - & 6q12q27(65,101,827-170,581,850), 18p11.32p11.21(179,428-14,989,963) \\
\hline 16 & - & $\begin{array}{l}\text { 6q16.1(97,717,588-97,817,598), 6q16.3(103,336,301-103,386,329) } \\
\text { 6q21(108,047,534-108,108,291) }\end{array}$ \\
\hline 17 & $\begin{array}{l}\text { 12(133BAC), 13q21.2q21.32(61,823,181-66,517,269), } \\
18 \mathrm{q} 23(73,359,922-77,875,127), 19 \mathrm{q} 13.1 \\
2 \mathrm{q} 13.2(38,161,572-42,940,785)\end{array}$ & 11q22.3(103,461,090-108,886,109) \\
\hline 18 & - & $\begin{array}{l}\text { 1p35.1p12(331,03,405-118,884,319), 3p26.3p26.1(968,957-4,677,964), } \\
\text { 8p23.3p23.1(81,046-11,941,743), 13q14.2q21.32(50,368-66,517), 13q31. } \\
\text { 3q34(93,702,860-114,120,603), 17p13.3p11.2(2,990,938-19,808,088), } \\
\text { 20q12q13(38,672,967-41,981,799) }\end{array}$ \\
\hline 19 & - & $\begin{array}{l}\text { 6q21(106,778,974-109,197,165), 13q14.2(50,368,875-50,546,925), } \\
22 \mathrm{q} 11.21(23,071,750-23,221,729)\end{array}$ \\
\hline 20 & $18 \mathrm{q} 21.31 \mathrm{q} 23(54,518,395-77,875,127)$ & $\begin{array}{l}\text { 7q31.1q34(107,642,872-141,079,377), 13q14.2q14.3(48,589,512-51,573,841), } \\
\text { 17p13.3p11.2(622,586-20,289,789) }\end{array}$ \\
\hline 21 & $\begin{array}{l}8 \mathrm{q} 24.21(128,508,282-129,064,398), 8 \mathrm{q} 24.2 \\
2 \mathrm{q} 24.3(134,803,426-146,227,426)\end{array}$ & 11q22.3(108,721,042-108,886,109), 14q32.33(106,196,305-107,146,073) \\
\hline 22 & - & $\begin{array}{l}\text { 13q14.2(50,368,875-50,952,073), 14q32.33(106,475,175-107,146,073), } \\
\text { 17p13.3p11.2(2,990,938-17,304,683) }\end{array}$ \\
\hline 23 & - & $11 \mathrm{q} 14.1 \mathrm{q} 22.3(85,061,154-108,886,109), 13 \mathrm{q} 14.2 \mathrm{q} 14.3(50,368,875-53,457,910)$ \\
\hline 24 & - & $13 q 14.2(50,368,875-50,952,073)$ \\
\hline 25 & - & $11 \mathrm{q} 22.3 \mathrm{q} 23.2(106,579,547-114,372,284), 13 \mathrm{q} 13.1(32,807,050-33,944,539)$ \\
\hline 26 & 12(172 BAC), 18(103 BAC), 19(75 BAC) & $13 \mathrm{q} 14.3(50,062,881-51,573,841)$ \\
\hline 27 & $2 \mathrm{p} 25.3 \mathrm{p} 12(235,263-77,339,292)$ & 11q14.1q23.2(78,965,050-115,755,274), 13q13.1(32,806,050-33,944,539) \\
\hline 28 & $12(172$ BAC), 18q22.1q22.3(66,676,923-68,777,622) & $\begin{array}{l}\text { 7p22p15.1(94,136-2,863,6921), 14q24.1q32.3(69,340,601-107,206,128), } \\
18 \mathrm{q} 21.2(52,781,830-53,160,754)\end{array}$ \\
\hline 29 & $18 \mathrm{q} 21.32 \mathrm{q} 22.3(53,348,598-69,890,646)$ & $\begin{array}{l}\text { 3p26.3p21.31(212,817-50,018,329), 7p22.3p15.1(94,136-28,636,921), } \\
\text { 9q22.33q34.3(101,849,491-140,997,194), 14q24.1q32.33(69,340,601-104,313,141), } \\
17 \mathrm{p} 13.1(7,495,272-7,741,642), 18 \mathrm{q} 21.2 \mathrm{q} 21.32(53,348,598-56,877,019)\end{array}$ \\
\hline 30 & - & $14 \mathrm{q} 24.1 \mathrm{q} 32.33(69,340,601-104,313,141)$ \\
\hline 31 & - & $11 \mathrm{q} 22.2 \mathrm{q} 24.1(102,281,372-122,793,220), 13 \mathrm{q} 13.3 \mathrm{q} 21.1(39,679,272-57,752,460)$ \\
\hline 32 & - & $6 \mathrm{q} 15 \mathrm{q} 25.3(88,252,488-158,934,425)$ \\
\hline 33 & - & $11 \mathrm{q} 14.2 \mathrm{q} 23.2(87,242,809-114,372,284)$ \\
\hline 34 & - & $13 \mathrm{q} 12.3 \mathrm{q} 22.1(31,951,813-74,404,613)$ \\
\hline 35 & $\begin{array}{l}\text { 5q34q35.3(164,820,779-180,124,695), 7q11.2 } \\
\text { 2q36.3(68,613,015-159,128,663), } \\
\text { 8q23.1q24.23(108,911,914-139,384,083) }\end{array}$ & $13 q 14.2 q 22.2(47,651,918-76,542,003), 17 q 25.3(76,125,623-80,781,537)$ \\
\hline 36 & $8 \mathrm{q} 21.3 \mathrm{q} 24.3(87,570,656-146,162,080)$ & $\begin{array}{l}\text { 6q21(108,047,534-108,540,335), 11q11q13.1( } 55,421,065-64,250,509) \\
11 \mathrm{q} 22.3 \mathrm{q} 25(106,579,547-132,886,220), 13 \mathrm{q} 12.11 \mathrm{q} 12.12(20,936,836-24,158,187) \\
13 \mathrm{q} 14.2 \mathrm{q} 14.3(48,999,921-52,514,204)\end{array}$ \\
\hline 37 & - & $13 \mathrm{q} 14.3(51,430,618-51,573,841)$ \\
\hline 38 & $12(143 \mathrm{BAC})$ & - \\
\hline 39 & $\begin{array}{l}\text { 2p25.3p16.3(1,633,836-49,658,363), 2p16. } \\
1 \mathrm{p} 14(59,105,825-64,822,652), \\
\text { 2p14(66,540,604-69,168,739), 2p12(76,319,546-80,955,167), } \\
\text { 4q28.2q35.2(130,877,638-190,782,221), 13q14. } \\
3 \mathrm{q} 21.32(53,285,718-65,890,658), \\
13 \mathrm{q} 22.2 \mathrm{q} 31.1(75,560,502-81,845,753), 13 \mathrm{q} 31 . \\
1 \mathrm{q} 34(82,012,303-114,912,704), \\
17 \mathrm{q} 21.31 \mathrm{q} 25.3(41,576,520-80,781,537)\end{array}$ & 17p13.3p11.2(1,618,017-18,169,260) \\
\hline 40 & (1) & $\begin{array}{l}\text { 9q21.13q34.12(76,396,515-133,480,644), 13q14.2(50,546,926-50,952,073), } \\
14 q 32.33(106,406,318-107,267,432), 17 \mathrm{p} 13.3 \mathrm{p} 11.2(622,586-17,304,683)\end{array}$ \\
\hline 41 & $2 \mathrm{p} 25.3 \mathrm{p} 12(2,271,126-68,307,621)$ & $13 \mathrm{q} 12.3 \mathrm{q} 22.1(31,150,628-31,789,825)$ \\
\hline 42 & - & $11 \mathrm{q} 14.1 \mathrm{q} 25(79,727,361-133,610,439)$ \\
\hline 43 & - & $13 q 14.2 q 14.3(4,707,348-50,925,202)$ \\
\hline 44 & - & $11 \mathrm{q} 22.3(102,611,654-107,782,670), 13 q 14.2(50,368,875-50,952,073)$ \\
\hline 45 & $8 q 21.3 q 24.23(90,050,966-137,910,471), 12(139 \mathrm{BAC})$ & 8p23.2-p12(2,317,216-31,967,062) \\
\hline 46 & - & $13 q 13.3 q 32.3(35,997,178-100,551,259)$ \\
\hline 47 & - & 13q14.2q14.3(48,894,899-50,454,033), 14q23q24.3(68,877,801-74,421,176) \\
\hline 48 & - & $11 \mathrm{q} 22.1 \mathrm{q} 23.3(10,172,554-116,704,710), 13 \mathrm{q} 14.2 \mathrm{q} 14.3(48,894,899-49,645,837)$ \\
\hline 49 & - & $13 q 14.2 q 14.3(48,467,994-52,285,963), 14 q 32.33(105,354,886-106,072,530)$ \\
\hline 50 & $\begin{array}{l}\text { 2p25.2(300,000-89,163,193), 8q22. } \\
3 \mathrm{q} 24 \cdot 3(103,802,271-103,959,652)\end{array}$ & $6 \mathrm{q} 16.3 \mathrm{q} 25.3(103,441,042-159,904,765)$ \\
\hline
\end{tabular}




\section{RESULTS}

The relevant clinical, cytogenetic, FISH and mutational status data of 50 CLL patients are summarized in Table 1. Cytogenetic results were obtained for 37 patients, and an abnormal karyotype was detected in 23 (46\%) patients. Translocations were detected and confirmed by FISH in 11 patients. A commercially available FISH panel revealed recurrent chromosomal changes in 47 (94\%) patients. In addition to already well-known changes (such as losses at $6 \mathrm{q}$ ( 7 patients/14\%), 11q22 (12 patients/2\%), $13 \mathrm{q} 14$ (33 patients/66\%), 17p13 (6 patients/12\%) and trisomy 12 (5 patients/10\%)), additional abnormalities were detected. Deletions or partial deletions and/or rearrangement involving $14 \mathrm{q} 32$ were found in 14 patients using FISH with a locus-specific probe for the $I g H$ gene, which covers both the constant and variable gene region. We confirmed deletion of the 5' IgVH flanking sequences in 10 cases, while 3' $\mathrm{IgH}$ deletion was detected in only 2 cases. Deletion of the whole $I g H$ gene was found in only 1 case, as well as rearrangement of the $I G H$ gene (Table 1). FISH targeted to non-routinely analyzed CLL regions was performed in selected cases to confirm and complete cytogenetic and arrayCGH findings (data not shown).

\section{Copy number aberrations revealed by arrayCGH}

ArrayCGH revealed copy number changes in 44 (88\%) out of 50 patients. We detected a total of 84 regions of loss and 34 regions of gain (Table 2). Using arrayCGH, we detected well-known aberrations as well as various novel imbalances. These new abnormalities were found in 14 patients and included losses of chromosomes $1 \mathrm{p}$ (no. 18), $3 \mathrm{p}$ (nos. 18 and 29), $7 \mathrm{p}$ (nos. 28 and 29), 7q (no. 20), 18 (no. 15), 8 p (nos.18 and 45), 9q (nos. 29 and 40), $15 q$ (no. 9), 17q (no. 35), 18p (no. 15), 18q (nos. 28 and 29), 20q (no. 18) and 22q (no. 19). Novel chromosomal gains included gains of chromosomes $5 \mathrm{q}$ (no. 35 ), $7 \mathrm{q}$ (no. 35), 17q (no. 39), 18q/18 (nos. 17, 20, 26, 28 and 29) and 19q/19 (nos. 17 and 27). In four cases (nos. 27, 39, 41 and 50), gains of chromosome $2 p$ were detected. In one (no. 39), the gained region was heterogeneous, as shown in Figure 1. The result of patient no. 50 was included in the data published previously ${ }^{24}$. The most frequent gains were detected on chromosomes 8q and 18q, each in 5 cases $(10 \%)$. These gains could be considered recurrent abnormalities. The most frequent losses were recognized on chromosome 6q ( 7 cases, $14 \%)$.

\section{Loss of 6q and determination of the smallest commonly deleted region}

Seven patients (nos. 5, 15, 16, 19, 32, 36 and 50) with $6 \mathrm{q}$ loss detected by FISH or arrayCGH were successfully subjected to $32 \mathrm{~K}$ tiling path chromosome 6 arrayCGH to more precisely define the smallest commonly deleted region (SCDR). We confirmed that the SCDR included 6q21q32 comprising a $1.4 \mathrm{Mb}$ region (Figure 2). The deleted region involved band q21 where a number of genes are located, among them the $F O X O 3 A$ gene.

\section{Comparison of FISH and arrayCGH results}

All 50 CLL samples had been previously analyzed using commercially available FISH probes mapping to routinely analyzed loci; 11q22 (ATM), centromeric region of chromosome 12, 13q14 and 17p13 (TP53). These results were compared to assess array performance versus interphase FISH. A total of $95 \%$ of the FISH findings were concordant with the arrayCGH results. Thirteen findings in 10 patients (nos. 4, 5, 9, 11, 14, 15, 27, 29, 44 and 45; Table 1,2) were discordant for various reasons. This discordance involved deletion of 11q22 containing the $A T M$ gene, which was detected in 12 cases by FISH but was not confirmed in 2 cases by arrayCGH. Trisomy of chromosome 12 was approved in all 5 FISH positive cases. Moreover in 1 patient (no. 5) partial trisomy of 12 q13q24 was detected only by arrayCGH and later confirmed by FISH with GLI (12q13) probe. Deletion of 13 q14 was confirmed by arrayCGH in only 29 out of the 33 cases detected by FISH with LSI D13S319 probe. On the other hand, arrayCGH revealed high heterogeneity of the deleted 13q14 region in other patients, which was displayed mainly in the results of oligonucleotide arrays (Table 2). Deletion of chromosome 17p13 detected by arrayCGH was concordant with FISH results in all examined cases.

\section{DISCUSSION}

We used 4 different arrayCGH platforms available in our laboratory during the study. This could cause inhomogeneous results due to platform-specific copy number variations. Gunnarsson et al. ${ }^{25}$ showed that all arrayCGH platforms concordantly detect large and known copy number alterations but diverge in detection of small ones. Therefore we took into account in this study, only abnormalities represented by at least three subsequent clones or copy number changes confirmed by FISH.

The arrayCGH revealed a total of 84 regions of loss and 34 regions of gain. We were able to detect copy number changes in regions with already well-known impact on CLL prognosis, such as deletions of the $A T M$ gene at $11 \mathrm{q} 22\left(\right.$ ref. $^{26,27}$ ), $m i R-15 a / 16-1$ and $R B 1$ genes at the $13 \mathrm{q} 14$ region $^{28-30}$, TP53 gene at $17 \mathrm{p} 13$ (ref. ${ }^{31,32}$ ) and trisomy 12 / gain of $12 q\left(\right.$ ref. $\left.{ }^{33}\right)$. We also identified other recurrent imbalances such as gains of $2 p$ and $8 q 24$ and loss of $14 q 32$. In addition, we noticed a large number of non-recurrent imbalances, most of them as new unbalanced changes (Table 2). Our results indicate that the genomic instability of CLL is higher than previously thought. We suspect that regions of recurrent chromosomal imbalances could result in altered expression of genes contributing to the pathogenesis of CLL but this was not aim of our study.

The frequent gain of the 2p14-p25 sequences detected by arrayCGH in 4 patients is of particular interest. These findings were confirmed by FISH with the LSI ALK, LSI NMYC, CEP 2 and REL-specific BAC probes. Our research team published finding $2 p$ gains in another cohort of CLL cases $^{24}$, and its prognostic significance was re- 


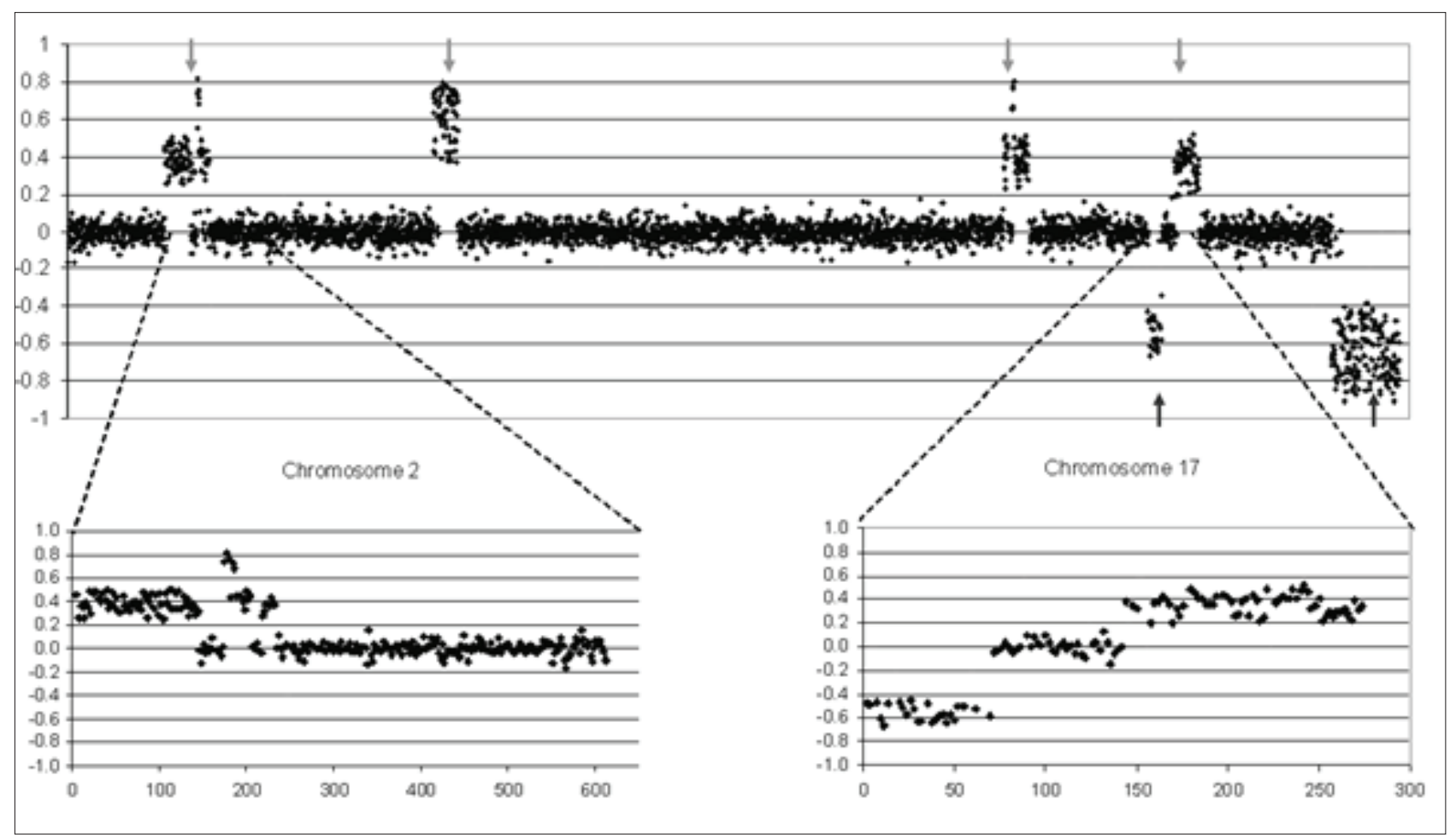

Fig. 1. Example of arrayCGH result in patient no. 39.

ArrayCGH profile is showing $\log 2$ ratios of $\mathrm{Cy} 5 / \mathrm{Cy} 3$ signal intensities along all chromosomes, from chromosome 1 to chromosome $\mathrm{X}$, from the left to the right side, respectively. ArrayCGH revealed gains of regions $2 \mathrm{p} 25.3 \mathrm{p} 16.3,2 \mathrm{p} 16.1 \mathrm{p} 14,2 \mathrm{p} 14,2 \mathrm{p} 12$, 4q28.2q35.2, 13q21.1q21.32, 13q22.2q31.1, 13q31.1q34, 17q21.31q25.3 (upper grey arrows) and losses of 17p13.3p11.2 region and chromosome X (due to sex-mismatch control) (lower black arrows). Lower part of figure is displaying detailed profiles of chromosome 2, note the 4 different regions of $2 \mathrm{p}$ gain, and chromosome 17 lost and gained regions.

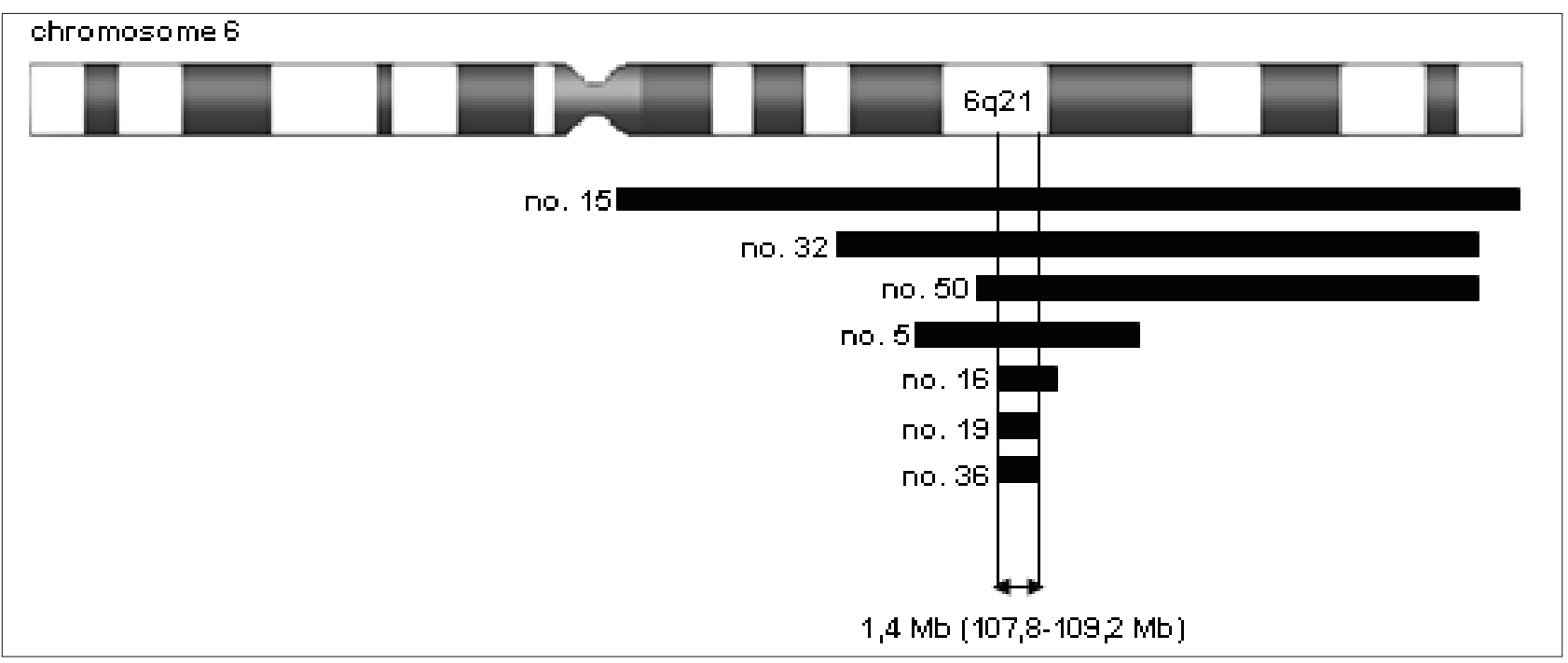

Fig. 2. Schematic summary of $32 \mathrm{~K}$ tiling path chromosome 6 arrayCGH results.

Black bars are representing deleted regions along chromosome $6 \mathrm{q}$ in 7 patients. The established SCDR covered 1,4 Mb at $6 \mathrm{q} 21$ (107,8-109,2 Mb).

cently confirmed by Chapiro et al. ${ }^{34}$. In 3 patients, the $2 p$ gain was accompanied by other cytogenetic abnormalities (nos. 27, 39 and 50) while another only had a 13q deletion (no. 41). The heterogeneity of the gained region is shown in Fig. 1, case no. 39. According to the literature ${ }^{3,35,36}$, a gain of $2 p$ sequences occurs in 7-17\% of CLL. This aberration is associated with a poor prognosis and could play an important role in the pathogenesis of the disease. For this reason, we again suggest that 2 p probes (e.g., NMYC or ALK) are included in the routine FISH investigation of CLL ( ref. $^{24}$ ).

Gains of the $8 \mathrm{q}$ region detected in 5 patients, 4 with an unmutated $\operatorname{IgVH}$ pattern, involved region q24 with the location of the CMYC gene. This finding was confirmed 
by FISH with the LSI MYC probe. The $8 \mathrm{q}$ gain would have remained undetected in 4 out of 5 patients if the conventional cytogenetics and routine FISH panel had alone been used.

The loss of $14 \mathrm{q} 32$ sequences was detected by FISH in 13 cases, whereas arrayCGH recognized this aberration in only 6 of these. This was probably caused by the already well-known presence of polymorphic sequences in this region. Losses in the $I G H$ region presumed to represent deletions occurring during the physiological I $\mathrm{H}$ gene rearrangement within B-cell development and is not associated with the pathogenesis of CLL (ref. ${ }^{37}$ ). On the other hand, the detected deletions tend to associate with additional chromosomal changes, such as trisomy 12 and $I g V H$ unmutated status ${ }^{38}$. Both cases with $3^{\prime} \mathrm{IgH}$ deletion were unmutated, as well as 7 out of 10 cases with 5 ' $\mathrm{IgVH}$ deletion, and in all cases additional changes were observed. A constant loss of $6 \mathrm{q}$ sequences, confirmed by FISH with the ON MM 6q21/15q22 probe, was detected by arrayCGH in 7 CLL patients. All these patients showed an unmutated $I g V H$ pattern related to a poor prognosis. In two patients, $\operatorname{del}(6)(q)$ was detected as the sole aberration; in the remaining five patients, $\operatorname{del}(6)$ (q) was accompanied by other molecular cytogenetic abnormalities, such as deletions of 11q22, 13q14, 17p13 and other regions. These findings are in agreement with published data that classify $\operatorname{del}(6)(\mathrm{q})$ as an adverse prognostic factor in lymphoid malignancies ${ }^{39,40}$. The smallest commonly deleted region at $6 \mathrm{q}$ established in 7 patients was defined by $32 \mathrm{~K}$ tiling path chromosome 6 arrayCGH and covered $1.4 \mathrm{Mb}$ at $6 \mathrm{q} 21(107.8-109.2 \mathrm{Mb})$. This region includes only one $F O X O 3 A$ gene previously reported as a gene that plays a role in B-cell activation, differentiation and pathogenesis ${ }^{40}$. The other genes with the same role in CLL, such as CCNC, CD24, PRDM1 located at 6q21 $(106.5 \mathrm{Mb})\left(\right.$ ref. $\left.^{41-44}\right)$, were excluded from consideration in our patients because they are located outside of our established SCDR.

We also suggest that an unknown tumor suppressor gene could be present within this region. Expression profiling ${ }^{45}$ may shed light on the events connected with $6 \mathrm{q} 21$ deletions as well as on other copy number changes in patients with B-CLL and their role in the malignancy.

Six out of 50 analyzed patients showed a balanced karyotype in arrayCGH. In 3 patients, this finding correlated with normal cytogenetic and FISH results. In 2 patients, only FISH detected deletions of 13q14, 11q22 and 5' $I G H$. We presume that these findings are either below the detection level of the arrayCGH technique (22\%) or that the 2 patients who escaped arrayCGH analysis have a deletion of 13 q14 of a very small size, which was not depicted by arrayCGH platform (Leiden, Netherlands) due to absence of BAC referring to this approximately 100 $\mathrm{kb}$ large region. A balanced chromosomal translocation $\mathrm{t}(3 ; 14)$ with rearrangement of the $\operatorname{Ig} H$ gene was found in 1 patient with an arrayCGH-balanced profile.

Our findings are in concordance with findings of other groups $^{36,46-49}$.

\section{CONCLUSIONS}

In summary, the $\mathrm{CGH}$ array proved to be a reliable tool for detecting quantitative genomic aberrations that escape cytogenetic and routine FISH analyses in CLL. It also helped to precisely map the breakpoints of unbalanced regions but failed to identify aberrations in samples with small deletion in region not presented on the platform and samples with low involvement of pathological cells. The detection limit of our arrayCGH platforms was $22 \%$ of abnormal cells in analyzed sample.

Cytogenetic examination of the studied CLL patients revealed chromosomal abnormalities in 23 (46\%) patients, whereas FISH analysis of six CLL monitored loci depicted 77 aberrations in 47 (94\%) patients. ArrayCGH, however, identified 118 genomic imbalances in 44 (88\%) patients, including new aberrations in 14 patients which had not been detected by FISH or cytogenetics. These results suggest that a subset of potentially significant genomic alterations in CLL is being missed by using only cytogenetics and FISH. Without conventional cytogenetics and FISH, however, it would not be possible to resolve complex karyotypes, balanced translocations and approximately $5 \%$ of abnormalities would be missed due to limits of arrayCGH.

In conclusion, we propose a combination of conventional cytogenetics, FISH and arrayCGH as a necessary tool to unravel the molecular karyotype of CLL as it is an important indicator of disease prognosis. Further detailed analysis of aberrant regions detected by arrayCGH, and complemented by cytogenetic and molecular findings as well as clinical parameters, could be of help in identification of new biomarkers in CLL with a potential prognostic value.

\section{ACKNOWLEDGEMENTS}

This work was supported by grants from the Czech Ministry of Health (NR9484-3) and the Czech Ministry of Education, Youth and Sports (MSM 6198959205).

We would like to thank the Wellcome Trust Sanger Institute for the BAC clone supply and Dr. Karoly Szuhai (Leiden University Medical Center, Leiden, Netherlands) and Paul Van Hummelen (VIB MicroArray Facility, Catholic University Leuven, Leuven, Belgium) for generating the arrayCGH slides.

\section{REFERENCES}

1. Rai KR, Sawitsky A, Cronkite EP, Chanana AD, Levy RN, Pasternack BS. Clinical staging of chronic lymphocytic leukemia. Blood 1975;46:219-34.

2. Binet JL, Auquier A, Dighiero G, Chastang C, Piguet $H$, Goasguen J, Vaugier G, Potron G, Colona P, Oberling F, Thomas M, Tchernia G, Jacquillat $C$, Boivin $P$, Lesty $C$, Duault MT, Monconduit $M$, Belabbes S, Gremy F. A new prognostic classification of chronic lymphocytic leukemia derived from a multivariate survival analysis. Cancer 1981;48:198-206.

3. Döhner H, Stilgenbauer S, Benner A, Leupolt E, Kröber A, Bullinger 
L, Döhner K, Bentz M, Lichter P. Genomic aberrations and survival in chronic lymphocytic leukamia. N Engl J Med 2000;343:1910-6.

4. Fegan C, Robinson H, Thompson P, Whittaker JA, White D. Karyotypic evolution in CLL: Identification of a new sub-group of patients with deletions of $11 \mathrm{q}$ and advanced or progressive disease. Leukemia 1995;9:2003-8.

5. Neilson JR, Auer R, White D, Bienz N, Waters JJ, Whittaker JA, Milligan DW, Fegan CD. Deletions at 11q identify a subset of patients with typical CLL who show consistent disease progression and reduced survival. Leukemia 1997;11:1929-32.

6. Geisler CH, Philip P, Christensen BE, Hou-Jensen K, Pedersen NT, Jensen OM, Thorling K, Andersen E, Birgens HS, Drivsholm A, Ellegaard J, Larsen JK, Plesner T, Brown P, Andersen PK, Hansen MM. In B-cell chronic lymphocytic leukaemia chromosome 17 abnormalities and not trisomy 12 are the single most important cytogenetic abnormalities for the prognosis: A cytogenetic and immunophenotypic study of 480 unselected newly diagnosed patients. Leuk Res 1997;21:1011-23.

7. Carney DA, Wierda WG. Genetics and molecular biology of chronic lymphocytic leukemia. Curr Treat Options Oncol 2005;6:215-25.

8. Stilgenbauer S, Dohner H. Campath-1H-induced komplete remission of chronic lymphocytic leukemia despote p53 mutation and resistence to chemotherapy. N Engl J Med 2002;347:452-3.

9. Caballero D, Garcia-Marco JA, Martino R, Mateos V, Ribera JM, Sarrá J, León A, Sanz G, de la Serna J, Cabrera R, González M, Sierra J, San Miguel J. Allogeneic transplant with reduced intensity conditions may overcome the poor prognosis of B-cell chronic lymphocytic leukemia with unmutated imunoglobulin variable haevy-chain gene and chromosomal abnormalities (11q-and 17p-). Clin Cancer Res 2005;11:7757-63.

10. Gahrton G, Robèrt KH, Friberg K, Zech L, Bird AG. Extra chromosome 12 in chronic lymphocytic leukaemia. Lancet 1980;1:146-7.

11. Matutes E, Oscier D, Garcia-Marco J, Ellis J, Copplestone A, Gillingham R, Hamblin T, Lens D, Swansbury GJ, Catovsky D. Trisomy 12 defines a group of CLL with atypical morphology: correlation between cytogenetic, clinical and laboratory features in 544 patients. $\mathrm{Br} J$ Haematol 1996;92:382-8.

12. Calin GA, Croce CM. Genomics of chronic lymphocytic leukemia microRNAs as a new players with clinical significance. Semin Oncol 2006;33:167-73.

13. Juliusson G, Oscier DG, Fitchett M, Ross FM, Stockdill G, Mackie MJ Parker AC, Castoldi GL, Guneo A, Knuutila S. Prognostic subgroups in B-cell chronic lymphocytic leukemia defined by specific chromosomal abnormalities. N Engl J Med 1990;323:720-4.

14. Oscier DG, Stevens J, Hamblin TJ, Pickering RM, Lambert R, Fitchett M. Correlation of chromosome abnormalities with laboratory features and clinical course in B-cell chronic lymphocytic leukaemia. Br J Haematol 1990;76:352-8.

15. Juliusson G, Oscier D, Gahrton G. For the International Working Party on Chromosomes in CLL (IWCCLL). Cytogenetic findings and survival in B-cell chronic lymphocytic leukemia. Second IWCCLL compilation of data on 662 patients. Leuk Lymphoma 1991;5:21-5.

16. Cuneo A, Rigolin GM, Bigoni R, De Angeli C, Veronese A, Cavazzini F, Bardi A, Roberti MG, Tammiso E, Agostini P, Ciccone M, Della Porta M, Tieghi A, Cavazzini L, Negrini M, Castoldi G. Chronic lymphocytic leukemia with $6 \mathrm{q}$ - shows distinct hematological features and intermediate prognosis. Leukemia 2004;18:476-83.

17. Nowakowski GS, Dewald GW, Hoyer JD, Paternoster SF, Stockero KJ, Fink SR, Smoley SA, Remstein ED, Phyliky RL, Call TG, Shanafelt TD, Kay NE, Zent CS. Interphase fluorescence in situ hybridization with an IGH probe is important in the evaluation of patients with a clinical diagnosis of chronic lymphocytic leukaemia. $\mathrm{Br} J$ Haematol 2005;130:36-42.

18. Dyer MJ, Oscier DG. The configuration of the immunoglobulin genes in B cell chronic lymphocytic leukemia. Leukemia 2002;16:973-84.

19. Cavazzini F, Hernandez JA, Gozzetti A, Russo Rossi A, De Angeli C, Tiseo R, Bardi A, Tammiso E, Crupi R, Lenoci MP, Forconi F, Lauria F, Marasca R, Maffei R, Torelli G, Gonzalez M, Martin-Jimenez P, Maria Hernandez J, Rigolin GM, Cuneo A. Chromosome 14q32 translocations involving the immunoglobulin heavy chain locus in chronic lymphocytic leukaemia identify a disease subset with poor prognosis. Br J Haematol 2008;142:529-37.

20. Gunn SR, Mohammed MS, Gorre ME, Cotter PD, Kim J, Bahler DW, Preobrazhensky SN, Higgins RA, Bolla AR, Ismail SH, de Jong $D_{\text {, }}$
Eldering E, van Oers MH, Mellink CH, Keating MJ, Schlette EJ, Abruzzo LV, Robetorye RS. Whole-Genome Scanning by Array Comparative Genomic Hybridization as a Clinical Tool for Risk Assessment in Chronic Lymphocytic Leukemia. J Mol Diagn 2008;10:442-51.

21. Fiegler $H$, Carr $P$, Douglas EJ, Burford DC, Hunt $S$, Scott $C E$, Smith J, Vetrie D, Gorman P, Tomlinson IP, Carter NP. DNA microarrays for comparative genomic hybridization based on DOP-PCR amplification of BAC and PAC clones. Genes Chromosomes Cancer 2003;36:361-74.

22 Vermeesch JR, Melotte C, Froyen G, Van Vooren S, Dutta B, Maas N, Vermeulen S, Menten B, Speleman F, De Moor B, Van Hummelen P, Marynen P, Fryns JP, Devriendt K. Molecular karyotyping: array CGH quality criteria for constitutional genetic diagnosis. J Histochem Cytochem 2005;53:413-22.

23. Knijnenburg J, Szuhai K, Giltay J, Molenaar L, Sloos W, Poot M, Tanke $\mathrm{HJ}$, Rosenberg C. Insights from genomic microarrays into structural chromosome rearrangements. Am J Med Genet 2005;132:36-40.

24. Jarosova M, Urbankova H, Plachy R, Papajik T, Holzerova $M$, Balcarkova J, Pikalova Z, Divoky V, Indrak K. Gain of chromosome $2 p$ in chronic lymphocytic leukemia: significant heterogeneity and a new recurrent dicentric rearrangement. Leuk Lymphoma 2010;51:304-13.

25. Gunnarsson R, Staaf J, Jansson M, Ottesen AM, Göransson H, Liljedah $\mathrm{U}$, Ralfkiaer $\mathrm{U}$, Mansouri M, Buhl AM, Smedby KE, Hjalgrim $\mathrm{H}$, Syvänen AC, Borg A, Isaksson A, Jurlander J, Juliusson G, Rosenquist R. Screening for copy-number alterations and loss of heterozygosity in chronic lymphocytic leukemia--a comparative study of four differently designed, high resolution microarray platforms. Genes Chromosomes Cancer 2008;47:697-711.

26. Schaffner C, Stilgenbauer S, Rappold GA, Döhner H, Lichter P. Somatic ATM mutations indicate a pathogenic role of ATM in B-cell chronic lymphocytic leukemia. Blood 1999;94:748-53.

27. Stankovic T, Weber P, Stewart G, Bedenham T, Murray J, Byrd PJ, Moss $\mathrm{PA}$, Taylor AM. Inactivation of ataxia telangiectasia mutated gene in B-cell chronic lymphocytic leukemia. Lancet 1999;353:26-9.

28. Bullrich F, Veronese ML, Kitada S, Jurlander J, Caligiuri MA, Reed JC, Croce CM. Minimal region of loss at 13q14 in B-cell chronic lymphocytic leukemia. Blood 1996;88:3109-15.

29. Kalachikov S, Migliazza A, Cayanis E, Fracchiolla NS, Bonaldo MF, Lawton L, Jelenc P, Ye X, Qu X, Chien M, Hauptschein R, Gaidano G, Vitolo U, Saglio G, Resegotti L, Brodjansky V, Yankovsky N, Zhang P, Soares MB, Russo J, Edelman IS, Efstratiadis A, Dalla-Favera R, Fischer SG. Cloning and gene mapping of the chromosome 13q14 region deleted in chronic lymphocytic leukemia. Genomics 1997;42:369-77.

30. Liu Y, Corcoran M, Rasool O, Ivanova G, Ibbotson R, Grandér $D$ lyengar A, Baranova A, Kashuba V, Merup M, Wu X, Gardiner A, Mullenbach R, Poltaraus A, Hultström AL, Juliusson G, Chapman R, Tiller M, Cotter F, Gahrton G, Yankovsky N, Zabarovsky E, Einhorn S, Oscier D. Cloning of two candidate tumor suppressor genes within a $10 \mathrm{~kb}$ region on chromosome 13q14, frequently deleted in chronic lymphocytic leukemia. Oncogene 1997;15:2463-73.

31. Döhner H, Fischer K, Bentz M, Hansen K, Benner A, Cabot G, Diehl D, Schlenk R, Coy J, Stilgenbauer S. p53 gene deletion predicts for poor survival and non-response to therapy with purine analogs in chronic B-cell leukemias. Blood 1995;85:1580-9.

32. El Rouby S, Bayona W, Pisharody SM, Newcomb EW. p53 mutations in B-cell chronic lymphocytic leukemia. Curr Top Microbiol Immunol 1992;182:313-7.

33. Dierlamm J, Wlodarska I, Michaux L, Vermeesch JR, Meeus P, Stul M, Criel A, Verhoef G, Thomas J, Delannoy A, Louwagie A, Cassiman JJ, Mecucci C, Hagemeijer A, Van den Berghe H. FISH identifies different types of duplications with $12 q 13-15$ as the commonly involved segment in B-cell lymphoproliferative malignancies characterized by partial trisomy 12. Genes Chromosomes Cancer 1997;20:155-66.

34. Chapiro E, Leporrier N, Radford-Weiss I, Bastard C, Mossafa H, Leroux D, Tigaud I, De Braekeleer M, Terré C, Brizard F, Callet-Bauchu E, Struski S, Veronese L, Fert-Ferrer S, Taviaux S, Lesty C, Davi F, MerleBéral H, Bernard OA, Sutton L, Raynaud SD, Nguyen-Khac F. Gain of the short arm of chromosome $2(2 p)$ is a frequent recurring chromosome aberration in untreated chronic lymphocytic leukemia (CLL) at advanced stages. Leuk Res 2010;34:63-8.

35. Haferlach C, Rauhut S, Merk S, Dicker F, Schnittger S, Kern W, Dugas $M$, Haferlach T. Gain of the short arm of chromosome 2 is a new recurrent chromosome aberration in CLL which is significantly as- 
sociated with an unmutated IgVH status and a complex aberrant karyotype and leads to a higher expression of genes located on $2 \mathrm{p}$. Blood 2007; (ASH Annual Meeting Abstracts) 110:2075.

36. Pfeifer D, Pantic M, Skatulla I, Rawluk J, Kreutz C, Martens UM, Fish $\mathrm{P}$, Timmer J, Veelken H. Genome-wide analysis of DNA copy number changes and LOH in CLL using high-density SNP arrays. Blood 2007;109:1202-10.

37. Maljaie SH, Brito-Babapulle V, Matutes E, Hiorns LR, De Schouwer PJ, Catovsky D. Expression of c-myc oncoprotein in chronic T cel leukaemias. Leukemia 1995;9:1694-9.

38. Wlodarska I, Matthews C, Veyt E, Pospisilova H, Catherwood MA Poulsen TS, Vanhentenrijk V, Ibbotson R, Vandenberghe P, Morris TCM, Alexander HD. Telomeric IGH losses detectable by FISH in chronic lymphocytic leukemia manifest somatic $\mathrm{VH}$ recombination events. J Mol Diagn 2007;9:47-54.

39. Reindl L, Bacher U, Dicker F, Alpermann T, Kern W, Schnittger S, Haferlach T, Haferlach C. Biological and clinical characterization of recurrent $14 \mathrm{q}$ deletions in CLL and other mature B-cell neoplasms. Br J Haematol 2010;151:25-36.

40. Stilgenbauer S, Bullinger L, Benner A, Wildenberger K, Bentz M, Döhner K, Ho AD, Lichter P, Döhner H. Incidence and clinical significance of $6 \mathrm{q}$ deletions in B-cell chronic lymphocytic leukemia. Leukemia 1999;13:1331-4.

41. Thelander EF, Ichimura K, Corcoran M, Barbany G, Nordgren A Heyman M, Berglund M, Mungall A, Rosenquist R, Collins VP, Grandér $D$, Larsson C, Lagercrantz S. Characterization of $6 q$ deletions in mature B cell lymphomas and childhood acute lymphoblastic leukemia. Leuk Lymphoma 2008;49:477-87.

42. Jackson A, Carrara P, Duke V, Sinclair P, Papaioannou M, Harrison CJ, Foroni L. Deletion of 6q16-q21 in human lymphoid malignancies: a mapping and deletion analysis. Cancer Res 2000;60:2775-9.
43. Zhang Y, Matthiesen $P$, Harder S, Siebert R, Castoldi G, Calasanz MJ, Wong KF, Rosenwald A, Ott G, Atkin NB, Schlegelberger B. A 3-cM commonly deleted region in 6q21 in leukemias and lymphomas delineated by fluorescence in situ hybridization. Genes Chromosomes Cancer 2000;27:52-8.

44. Tam W, Gomez M, Chadburn A, Lee JW, Chan WC, Knowles DM. Mutational analysis of PRDM1 indicates a tumor-suppressor role in diffuse large B-cell lymphomas. Blood 2006;107:4090-100.

45. Haslinger C, Schweifer N, Stilgenbauer S, Döhner H, Lichter P, Kraut $\mathrm{N}$, Stratowa C, Abseher R. Microarray gene expression profiling of B-cell chronic lymphocytic leukemia subgroups defined by genomic aberrations and VH mutation status. J Clin Oncol 2004;22:3937-49.

46. Schwaenen C, Nessling M, Wessendorf S, Salvi T, Wrobel G, Radlwimmer B, Kestler HA, Haslinger C, Stilgenbauer S, Döhner H, Bentz M, Lichter P. Automated array-based genomic profiling in chronic lymphocytic leukemia: Development of a clinical tool and discovery of recurrent genomic alterations. Proc Natl Acad Sci U S A 2004;101:1039-44.

47. Tyybakinoja A, Vilpo J, Knuutila S. High-resolution oligonucleotide array-CGH pinpoints genes involved in cryptic losses in chronic lymphocytic leukemia. Cytogenet Genome Res 2007;118:8-12.

48. Schultz RA, Delioukina M, Gaal K, Bedell V, Smith DD, Forman SJ, McDaniel LD, Ballif BC, Shaffer LG, Slovak ML. Evaluation of chronic lymphocytic leukemia by BAC-based microarray analysis. Molecular Cytogenetics 2011;4:4.

49. Gunnarsson R, Mansouri L, Isaksson A, Göransson H, Cahill N, Jansson M, Rasmussen M, Lundin J, Norin S, Buhl AM, Smedby KE, Hjalgrim H, Karlsson K, Jurlander J, Geisler C, Juliusson G, Rosenquist R. Array-based genomic screening at diagnosis and during follow-up in chronic lymphocytic leukemia. Haematologica 2011;96:1161-9. 